\title{
Intoxicação exógena acidental em crianças no estado da Bahia: 2013 a 2017
}

\author{
Accidental exogenous poisoning in children in the state of Bahia: 2013 to 2017
}

Envenenamiento exógeno accidental en niños en el estado de Bahia: 2013 a 2017

Kaique Vinicius da Cruz Santos Aguiar ${ }^{1 *}$, Rafaela da Cunha Cruz ${ }^{1}$, Rafaela Trabuco de Araújo Silva ${ }^{1}$, Alan Santos Bonfim¹.

\section{RESUMO}

Objetivo: Caracterizar o perfil epidemiológico dos casos de intoxicação exógena acidental em crianças de 0 a 14 anos, no estado da Bahia, entre os anos de 2013 e 2017. Métodos: Estudo epidemiológico retrospectivo exploratório de caráter descritivo, realizado através dos dados secundários do Sistema de Informação de Agravos de Notificação (SINAN). Resultados: Foram identificadas 2.494 notificações, onde a faixa etárias mais acometida foi a de1 a 4 anos, representando $70 \%(n=1740)$. O sexo masculino obteve a maior incidência com $53 \%(n=1325)$ e as mulheres corresponderam $47 \%(n=1168)$. Quanto a raça/cor, os indivíduos da cor parda estiveram em maior prevalência $45 \%(n=1134)$ e a notificação ignorada obtiveram $43 \%(n=1075)$. Os agentes tóxicos mais frequentes foram os medicamentos $38 \%(n=956)$ e os produtos de uso domiciliar $21 \%(n=520)$. Conclusão: Considerando os resultados encontrados, salienta-se a importância de intensificar as medidas preventivas, principalmente pelos profissionais da atenção primaria, ainda, é importante enfatizar a necessidade de investimento governamental e sanitário em medidas de controle e em centros de controle de intoxicações.

Palavras-chave: Intoxicação, Crianças, Medicamentos.

\section{ABSTRACT}

Objective: To characterize the epidemiological profile of cases of accidental exogenous poisoning in children aged 0 to 14 years, in the state of Bahia, between the years 2013 and 2017. Methods: Retrospective exploratory epidemiological study of a descriptive character, carried out using secondary data from the System Information System for Notifiable Diseases (SINAN). Results: 2,494 notifications were identified, where the age group most affected was 1 to 4 years, representing $70 \%(n=1740)$. Males had the highest incidence with $53 \%(n=1325)$ and women accounted for $47 \%(n=1168)$. As for race / color, brown individuals were $45 \%$ more prevalent $(n=1134)$ and ignored notification obtained $43 \%(n=1075)$. The most frequent toxic agents were medicines $38 \%(n=956)$ and household products $21 \%(n=520)$. Conclusion: Considering the results found, the importance of intensifying preventive measures is emphasized, mainly by primary care professionals. It is also important to emphasize the need for government and health investment in control measures and in poison control centers.

Key words: Intoxication, Children, Medicines.

\section{RESUMEN}

Objetivo: Caracterizar el perfil epidemiológico de los casos de intoxicación exógena accidental en niños de 0 a 14 años, en el estado de Bahía, entre los años 2013 y 2017. Métodos: Estudio epidemiológico exploratorio retrospectivo de carácter descriptivo, realizado con datos secundarios del Sistema de Información para Enfermedades de Notificación (SINAN). Resultados: Se identificaron 2.494 notificaciones, donde el grupo de edad más afectado fue de 1 a 4 años, lo que representa el $70 \%(n=1740)$. Los hombres tuvieron la mayor incidencia con $53 \%(n=1325)$ y las mujeres representaron $47 \%(n=1168)$. En cuanto a la raza / color, los individuos marrones fueron $45 \%$ más prevalentes $(n=1134)$ y la notificación ignorada obtuvo $43 \%(n=1075)$. Los agentes tóxicos más frecuentes fueron medicamentos $38 \%(n=956)$ y productos para el hogar $21 \%(n=$ 520). Conclusión: Considerando los resultados encontrados, se enfatiza la importancia de intensificar las

${ }^{1}$ Faculdade Pitágoras de Feira de Santana, Feira de Santana - BA. *E-mail: kaique.vinicius30@hotmail.com SUBMETIDO EM: 3/2020 
medidas preventivas, principalmente por parte de los profesionales de atención primaria, y también es importante enfatizar la necesidad de inversión gubernamental y de salud en medidas de control y en centros de control de envenenamiento.

Palabras clave: Intoxicación, Niños, Medicamentos.

\section{INTRODUÇÃO}

As pessoas são constantemente expostas a uma grande variedade de produtos químicos de diversas formas e volumes, Ihes deixando vulneráveis a acidentes, a exemplo das intoxicações. As intoxicações estão entre os casos de lesões não intencionais que são responsáveis por um expressivo número de atendimentos em hospitais de países de diferentes continentes, representando um problema de saúde pública (VILAÇA L e CARDOSO P, 2014). Estima-se que, em de 2012, cerca de 193.460 pessoas morreram em todo o mundo por envenenamento não intencional (WHO, 2018). Intoxicação exógena ou envenenamento é o conjunto de efeitos nocivos representados por manifestações clínicas ou laboratoriais que revelam o desequilíbrio orgânico produzido pela interação de um ou mais agentes tóxicos com o sistema biológico (BRASIL, 2017).

As lesões na infância representam cerca de $40 \%$ de todas as mortes de crianças que, muitas vezes, podem ser evitadas ou controladas (WHO, 2008). Estima-se que 345.814 pessoas morreram em todo o mundo por envenenamento acidental em 2004, sendo que 13\% desses óbitos eram crianças e jovens menores de 20 anos (WHO, 2008). Nos Estados Unidos em 2012, tiveram mais de 2,2 milhões de exposições humanas a toxinas, com 93,7\% ocorrendo em uma residência e 48,4\% entre crianças menores de 6 anos (MADDEN M, 2015).

A intoxicação exógena aguda é considerada uma das emergências médicas mais comuns na população pediátrica, onde na maioria dos casos está relacionado com exposição a produtos domésticos tóxicos (ALRUWAILI ND, et al., 2019). As crianças estão particularmente em risco a este agravo, pelo seu comportamento curioso e exploratório, e atividades mão a boca, onde brincar perto do chão aumenta a exposição a toxinas (SOWMYA S, et al., 2014).

Os principais determinantes para a exposição de crianças e adolescentes a produtos tóxicos envolve armazenamento incorreto de produtos tóxicos e supervisão inadequada (SIQUEIRA KM, et al., 2008). O ambiente domiciliar apresenta uma grande variedade de agentes que quando não armazenados ou utilizados corretamente representam risco para intoxicação, onde a via oral é a principal via de intoxicação infantil (BRITO JG, MARTINS CBG, 2015).

Considerando a vulnerabilidade de crianças para intoxicação exógena, o problema de saúde pública que isso cabe e o impacto familiar, social, econômico e cultural gerado pela perda humana prematura resultada de envenenamentos na infância, é de fundamental importância conhecer as características epidemiológicas dos casos, pois irá possibilitar identificar o padrão de exposição para o subsidio de diretrizes de gerenciamento e políticas para esse agravo, além de favorecer no planejamento de medidas evitáveis, o que torna este estudo relevante. Este estudo tem como objetivo caracterizar o perfil epidemiológico dos casos de intoxicação exógena acidental em crianças de 0 a 14 anos, no estado da Bahia, entre anos de 2013 e 2017.

\section{MÉTODOS}

Trata-se de um estudo epidemiológico retrospectivo exploratório de caráter descritivo, realizado através dos dados secundários do Sistema de Informação de Agravos de Notificação (SINAN) disponibilizados no sitio eletrônico do Departamento de Informática do Sistema Único de Saúde (DATASUS), onde foram estudadas as notificações de intoxicação exógena acidental em crianças na faixa etária de 0 a 14 anos do estado da Bahia, no período de 2013 a 2017.

O SINAN é um sistema que coleta e fornece dados gerados pelo Sistema de Vigilância Epidemiológica brasileiro sobre notificação e investigação de doenças e agravos da lista nacional de doenças de notificação compulsória. O DATASUS permite análises da situação sanitária contribuindo para tomadas de decisão baseadas em evidências e subsídio ou manutenção de programas de saúde. 
As variáveis estudadas foram faixa etária de 0 a 14 anos, cor/raça, sexo e tipo de agente tóxico. As informações foram coletadas no dia 21 de dezembro de 2019. E o estudo adotou análise estatística descritiva, utilizando como ferramenta o Software Microsoft Exce ${ }^{\circledR}$. Por se tratar de dados disponíveis em banco de domínio público, o presente estudo não necessitou da análise do Comitê de Ética.

\section{RESULTADOS}

Na Bahia, entre os anos de 2013 a 2017 houveram 18.598 casos de intoxicação exógena, onde 20,2\%, ou seja, 3.769 casos corresponderam à circunstância acidental. As intoxicações acidentais entre a população de 0 a 14 anos foi responsável por 2.494 notificações, representando $13,4 \%$ de todos os casos de intoxicação exógena e $66.1 \%$ dos casos da circunstância acidental.

Na distribuição etária, verificou-se uma maior frequência de intoxicações entre a faixa etária de 1 a 4 anos responsável por $70 \%(n=1740)$ dos casos, seguido da faixa etária de 5 a 9 anos que representou $15 \%(n=369)$. Quanto ao sexo, os homens obtiveram a maior incidência com $53 \%(n=1325)$ das notificações e as mulheres obtiveram $47 \%(n=1168)$. Estratificando a faixa etária pelo sexo, constatou-se que as crianças de 1 a 4 anos do sexo masculino estavam envolvidos na maioria dos casos de intoxicação, correspondendo $n=958$ ao que diz $38 \%$, sendo que a mesma faixa etária do sexo feminino ficou na segunda posição com $31 \%(n=781)$ das notificações. As crianças de 5 a 9 anos do sexo masculino representaram $8 \%(n=188)$ dos casos (Tabela 1).

Tabela 1 - Distribuição das intoxicações exógenas acidentais segundo sexo e faixa etária de 0 a 14 anos, n=2.494. Estado da Bahia, Brasil, 2013 - 2017.

\begin{tabular}{ccccccc}
\hline Variável & \multicolumn{2}{c}{ Masculino } & \multicolumn{2}{c}{ Feminino } & \multicolumn{2}{c}{ Total } \\
\hline & $\mathbf{N}$ & $\%$ & $\mathbf{N}$ & $\%$ & $\mathbf{N}$ & $\%$ \\
\hline Faixa etária & & & & & & \\
\hline$<1$ Ano & 100 & 4 & 101 & 4 & 201 & 8 \\
$1-4$ & 958 & 38 & 781 & 31 & 1740 & 70 \\
$5-9$ & 188 & 8 & 181 & 7 & 369 & 15 \\
$10-14$ & 79 & 3 & 105 & 4 & 184 & 7 \\
\hline Total & 1325 & 53 & 1168 & 47 & 2494 & 100 \\
\hline
\end{tabular}

Fonte: Aguiar KVCS, et. al, 2020; dados extraídos de DATASUS/ SINAN.

A raça/cor parda esteve em maior prevalência entre as intoxicações, responsável por 1134 casos, ou seja, $45 \%$. Entretanto, cabe ressaltar que uma grande parte da variável raça/cor teve a informação ignorada, deixando $43 \%(n=1.075)$ registros sem essa informação. A raça/cor branca correspondeu 155 e a preta 120 notificações, representando $6 \%$ e $5 \%$ das notificações respectivamente (Tabela 2).

Tabela 2 - Perfil das intoxicações exógenas acidentais entre a faixa etária de 0 a 14 anos segundo raça/cor, n=2.494. Estado da Bahia, Brasil, 2013 - 2017.

\begin{tabular}{ccc}
\hline Variável & N & $\%$ \\
\hline Raça & & \\
\hline Branca & 155 & 6 \\
Preta & 120 & 5 \\
Amarela & 3 & 0 \\
Parda & 1134 & 45 \\
Indígena & 7 & 0 \\
Ignorado & 1075 & 43 \\
\hline Total & 2494 & 100 \\
\hline
\end{tabular}

Fonte: Aguiar KVCS, et al., 2020; dados extraídos de DATASUS/ SINAN. 
Com relação ao agente tóxico envolvido, constatou-se que os medicamentos estiveram em maior prevalência, responsável por $38 \%$ das intoxicações, seguido dos produtos de uso domiciliar que representaram 21\% dos casos. Em números absolutos 956 e 520 casos respectivamente. As causas consideradas ignoradas também corresponderam uma parcela significante, $11 \%$ das notificações. As intoxicações causadas por plantas tóxicas condisseram com 119 registros, ou seja $5 \%$. E os raticidas e produtos químicos ambos estiveram envolvidos com $4 \%$ das intoxicações (Tabela 3 ).

Tabela 3 - Perfil das intoxicações exógenas acidentais segundo agente etiológico, $\mathrm{n}=2.494$. Estado da Bahia, Brasil, $2013-2017$.

\begin{tabular}{ccc}
\hline Variável & N & $\%$ \\
\hline Agente etiológico & 956 & 38 \\
\hline Medicamento & 36 & 1 \\
Agrotóxico agrícola & 80 & 3 \\
Agrotóxico doméstico & 5 & 0 \\
Agrotóxico de saúde pública & 99 & 4 \\
Raticida & 64 & 3 \\
Produto veterinário & 520 & 21 \\
Produto de uso domiciliar & 82 & 3 \\
Cosmético & 111 & 4 \\
Produto químico & 6 & 0 \\
Metal & 3 & 0 \\
Abuso de drogas & 119 & 5 \\
Planta tóxica & 20 & 1 \\
Alimento e bebida & 116 & 5 \\
Outro & 277 & 11 \\
Ignorado & 2494 & 100 \\
\hline Total &
\end{tabular}

Fonte: Aguiar KVCS, et al., 2020; dados extraídos de DATASUS/ SINAN.

\section{DISCUSSÃO}

Os resultados deste estudo demonstraram que os casos de intoxicação exógena em crianças menores de 14 anos durante o espaço de cinco anos no estado da Bahia, estiveram em grande incidência, responsável por $13,4 \%$ de todos os casos de intoxicação exógenas registrados no SINAN e correspondeu $66.1 \%$ dos casos da circunstância acidental.

O estudo evidenciou o predomínio do grupo de crianças de 1 a 4 anos do sexo masculino, qual obteve $38 \%$ de prevalência. Os homens representaram $53 \%$ dos casos, semelhante com o estudo recente de Vilaça $\mathrm{L}$, et al (2020) que estudou o perfil dos atendimentos de crianças e adolescentes vítimas de intoxicações exógenas acidentais em um serviço de toxicologia de referência de um hospital público, onde o sexo masculino esteve envolvido em $55 \%$ das ocorrências. Igualmente constatado pela pesquisa de Domingos SM, et al. (2016), onde o sexo masculino apresentou maior número de internações, em todas as faixas etárias.

Em relação ao grupo etário, os resultados do nosso estudo são equivalentes a várias evidências encontradas em diversos locais do Brasil, onde há maior incidência na população de 1 a 4 anos, demonstrando que esta faixa etária está mais vulnerável as intoxicações (TAVARES EO, et al., 2013; OLIVEIRA FFS e SUCHARA EA, 2014; DOMINGOS SM, et al., 2016; VILAÇA L, et al., 2020). Ainda, Lacerda EMCB, et al. (2018) e Guimarães TRA, et al. (2019) estudaram as intoxicações exógenas considerando todas as idades, onde a idade de 1 a 4 anos ocupou a segundo posição das estatísticas, representando 26 e $26,13 \%$ respectivamente, ficando atrás apenas do grupo de 20 a 39 anos, isso sugere que mesmo comparado com todas as faixa etárias, as ocorrências entre o população de 1 a 4 anos ainda está em importante posição de prevalência. 
Este achado pode estar relacionado a fatores do próprio desenvolvimento infantil, levando em consideração que nesta faixa etária a criança se tona mais hábil, consegue abrir com mais facilidade recipientes e com a capacidade de deambular permite a exploração dos ambientes de forma íntima contribuindo para amplo acesso a locais de exposição a produtos domiciliares, medicamentos e outros objetos ameaçadores, levando tudo que encontram à boca.

O público de 10 a 14 anos tiveram menos envolvidos entre as intoxicações acidentais, isso sugere que com o aumento da idade, diminua o risco para este agravo. Conforme o avançar a idade diminui o risco das intoxicações exógenas acidentais, pois há maior compreensão do que é certo ou errado e do que oferece risco ou não(SILVA TJ e OLIVEIRA VB, 2018).Porém outros tipos de acidentes tendem a acontecer, pois à medida que as crianças crescem ficam mais dependentes, os deixando mais expostos aos fatores ambientais, o que contribuem para que diversos outros tipos de acidentes ocorram.

Quanto aos agentes etiológicos envolvidos, o medicamento foi o principal responsável pelas intoxicações, presentes em $38 \%$ dos casos. Esse mesmo desfecho esteve presente em pesquisas nacionais e internacionais, sendo um problema de atenção mundial (MARTINS CBG, et al., 2006; AMORIM MLP, et al., 2017; MORADI M, et al., 2016; WHO, 2008; ALRUWAILI ND, et al., 2019).

Em um estudo feito no Hospital Universitário Regional de Maringá, os medicamentos foram responsáveis por $42,1 \%$ internações por intoxicação em crianças de 0 a 14 anos ocorrendo entre 2006 a 2011. E os dados coletados pelo Hospital João XXII de Belo Horizonte, obtiveram os medicamentos responsáveis por 36,5\% dos envenenamentos em crianças. Ambos os estudos apontaram que esse mesmo agente causador estava em locais de fácil acesso o que favorece a exposição das crianças aos agentes tóxicos (DOMINGOS SM, et al., 2016; VILAÇA L, et al., 2020).

Alguns fatores que podem contribuir para a intoxicação farmacológica acidental por crianças são: aumento do consumo de medicamentos pela sociedade, automedicação, armazenamento inapropriado dos medicamentos no domicílio e as diversidades de formas, tamanhos e cores dos fármacos leva a criança associar a doces. Domingos SM, et al. (2016) diz que também faz parte desses fatores o uso desses medicamentos diante das crianças, a oferta do medicamento prescrito à criança associando-o a balas e doces e o descuido no descarte desses produtos.

Um estudo realizado no Centro de Assistência Toxicológica do Ceará (CEATOX-CE), que investigou o perfil dos medicamentos indutores de intoxicação exógena em crianças atendidas no hospital, evidenciou os anticonvulsivantes responsáveis por $22,6 \%$ das internações, os bronco dilatadores por $10,3 \%$ e os complexos vitamínicos por 9,3\%. A somatória de usos diversos (analgésicos, antipiréticos, anti-hipertensivo, antimicótico...) responderam $53,4 \%$ das intoxicações medicamentosas na faixa etária de 0 a 9 anos. Esses são os tipos de medicamentos mais comuns em intoxicação acidental por crianças (ALCÂNTARA DA, et al., 2013).

No estado de Pernambuco, uma pesquisa também identificou os medicamentos como principais agentes de intoxicação entre crianças de 1 a 12 anos, responsáveis por $45,0 \%$ dos casos. Dentre eles os medicamentos do tipo psicotrópicos foram os mais notificados $(17,0 \%)$, seguidos dos analgésicos $(5,8 \%)$ e estimulantes de apetite (3,1\%) (AMORIM MLP, et al., 2017). No Piauí, o padrão das intoxicações medicamentosas entre 2007 e 2012, foi em crianças de 1á 4 anos, do sexo feminino, por circunstância acidental e os benzodiazepínicos foi a droga causadora mais comum (MAGALHÃES JV, et al., 2013).

Os produtos de uso domiciliares estão em segundo lugar nas estatísticas de agente tóxico desse estudo, presentes em $21 \%$ das intoxicações. O mesmo resultado se repetiu no estudo realizado por Vilaça $L$, et al (2020) de Minas Gerais, onde os produtos de uso domiciliar foi o segundo agente causador mais comuns entre as intoxicações ocorridas com crianças, ficado atrás apenas dos medicamentos. Esses produtos de uso domiciliares incluem produtos de limpeza como detergente, desinfetante, água sanitária, sabão e etc, e tem uma grande potencialidade tóxica.

Em um estudo semelhante realizado no Centro de Assistência Toxicológica de Pernambuco (CEATOX$\mathrm{PE})$, a água sanitária foi o produto mais notificado entre essa classe $(70,8 \%)$, seguidos do cloro e querosene ambos com 8,0\% (AMORIM MLP, et al., 2017). 
Na maioria das vezes os diversos agentes acusadores de intoxicações são fornecidos pelas indústrias com apresentação atraente para o público infantil. Vários produtos domésticos são líquidos coloridos, em alguns casos até comercializados em garrafas tipo "PET", vendidas sem rótulo de identificação, e com armazenamento muitas vezes inadequado no lar. Como os medicamentos, que também é apresentado diversas vezes coloridos, decoradas e alguns de sabor agradável que atraem a atenção das crianças (VILAÇA L, et al., 2020; SILVA TJ e OLIVEIRA VB, 2018).

Embora as intoxicações por abuso de drogas numericamente foram pouco expressivas, 3 notificações, é imprescindível a atenção para este agente causador, visto que ouve o consumo de drogas por crianças que representa um problema muito complexo, e que traz diversos riscos físicos, psicológicos e sociais para essas crianças.

No que se refere ao perfil da raça/cor dos casos de intoxicação exógena infantil acidental em crianças no Estado da Bahia, houve destaque para a cor parda. Embora esse achado é prejudicado levando em conta que $43 \%$ dessa informação foi ignorada, quase metade das notificações, assim representando um viés.

A questão associada à categoria parda, em particular, representa um dos maiores desafios das classificações sobre a origem étnico-racial no país. No entanto, não se deve deixar de lado a categoria morena que é muito comum nas definições dos brasileiros. O epíteto mais adequado para classificar a categoria intermediária entre brancos e pretos, encontra-se categorias parda. Pode se especular que as pessoas costumam definir a cor parda, porque ainda não tem uma identidade racial (MAIO MC et al.2005).

Observa-se que o percentual de ignorados, encontra-se muito próximo da cor parda, com $43 \%$ da população pesquisada. As pessoas ainda costumam achar irrelevante quanto sua descrição racial, nesses casos, seus responsáveis por estarem em situação muitas vezes de emergência, querem no primeiro momento a saúde de seus filhos e familiares, nessa hora passar por despercebido esse fator.

A residência é o local de maior ocorrência dos casos de intoxicações infantil acidentais, demonstrando que o próprio domicílio é um local de risco para as crianças, principalmente aquelas da faixa etária entre 0 a 4 anos. Além disso, outros fatores que contribuem para este agravo é a alta medicalização da sociedade, aumento do uso de produtos de limpeza doméstica ligado a presença desses produtos no domicílio, a falta de supervisão ou ocupação dos adultos com outras atividade e o armazenamento inadequado dos produtos tóxicos (TAVARES EO, et al., 2013).

A consequência de exposição a toxinas dependem de muitos fatores, incluindo idade, se o agente é farmacêutico ou não farmacêutico, quantidade de exposição, tempo de exposição, tempo desde a apresentação até a unidade de saúde e tempo de intervenção (ALRUWAILI ND, et al., 2019).E o tempo entre a ingestão e o atendimento é muito importante para a avaliação do risco envolvido e na definição de qual a melhor escolha terapêutica (OLIVEIRA FFS e SUCHARA EA, 2014).

A redução ou eliminação de fatores de risco, como a falta de vigilância dos cuidadores e o armazenamento inadequado dos produtos potencialmente tóxicos, possibilitariam a redução de em torno de 13 a $19 \%$ respectivamente de intoxicação acidental na infância (AMORIM MLP, et al., 2017,apudHAHN RC, et al., 2013).

Além disso, o armazenamento seguro de plantas e disponibilidade do número de centros de controle de intoxicações traz resultados positivos na prevenção de intoxicações acidentais infantis. As ações educativas sobre melhorias no armazenamento seguro de medicamentos e produtos domésticos também agrega resultados positivos, especialmente aqueles que oferecem gratuitamente ou a baixo custo cadeados para armários ou adesivos com telefone dos centros de controle de intoxicações (LADEIRA RM, et al., 2016).

A intensificação de campanhas de prevenção de acidentes toxicológicos na infância é uma medida eficaz para a diminuição das ocorrências e para a aquisição de novos comportamentos que favoreçam para a manutenção de baixos níveis de intoxicação. Da mesma forma, também é imprescindível o empenho do governo em subsidiar e cumprir medidas como o uso de embalagem especial de proteção à criança, com tampa inviolável e disponibilização de doses fracionadas para extinguir as "farmácias caseiras" (TAVARES EO, et al., 2013). 
Para a aquisição desses novos comportamentos é preciso orientar pais e cuidadores sobre acondicionamento de agentes tóxicos e vigilância da família com conscientização dos riscos do ambiente doméstico (TAVARES EO, et al., 2013). Além disso, é de fundamental o ensino sobre o uso de medicamentos, importante que as orientações sejam realizadas desde os primeiros anos de vida, mostrando a importância e o perigo que os medicamentos podem trazer (SILVA TJ e OLIVEIRA VB, 2018).

Segundo Silva MF, et al. (2017), existe pouca atuação dos profissionais de saúde no que se trata de orientações sobre medidas de prevenção de acidentes domésticos, tanto anteriores como após sua ocorrência. Essas orientações são fundamentais na prevenção de acidentes e minimização das sequelas que deles podem advir. Os profissionais de saúde têm uma grande responsabilidade na educação e alerta da família para a prevenção de acidentes, pois estes lhe conferem uma grande credibilidade. No âmbito da atenção básica, no momento da consulta pediátrica compreende em um espaço privilegiado para execução de tais intervenções educacionais, além de fazer a notificação adequada quando ocorrer algum caso de intoxicação externa.

O enfermeiro, como integrante da equipe multiprofissional de assistência à saúde, desempenha um importante papel na educação em saúde e nas medidas de prevenção das intoxicações na infância. Pode atuar subsidiando a implementação de programas nas escolas, nas creches, nas comunidades e na Estratégia Saúde da Família; no tratamento, realizando um plano de assistência de enfermagem a criança intoxicada e orientando os pais e/ou responsáveis quanto ao atendimento domiciliar da intoxicação (LOURENÇO J, et al., 2008).

De acordo com os desfechos desse estudo, salienta a necessidade de investimento e intensificação em medidas de prevenção. Foram identificadas no estudo porcentagens significativas do desfecho ignorado, isso por erro do sistema de notificações em saúde ou preenchimento dos dados primários, além disso, o SINAN não fornece outras informações relevantes, como os tipos de medicamentos, os tipos de produtos de limpeza e o local que ocorreu a intoxicação. Com isso, é sugerido novos estudos que possam ampliar as tendências.

\section{CONCLUSÃO}

O estudo conseguiu identificar o perfil dos casos de intoxicação exógena acidental em crianças de 0 a 14 anos, no estado da Bahia, no período de cinco anos. Identificou que as crianças de 1 a 4 anos do sexo masculino são mais vulneráveis as intoxicações acidentais, além disso, os indivíduos da cor parda também estiveram prevalentes entre os casos. Os medicamentos e os produtos de uso domiciliares foram os principais agentes causadores das intoxicações. Considerando os resultados encontrados neste estudo e levando em conta que muitas vezes esse agravo pode ser evitável, salienta-se a importância de intensificar as medidas preventivas, principalmente pelos profissionais da atenção primaria, através de orientação e educação em saúde, ainda, é importante enfatizar a necessidade de investimento governamental e sanitário em medidas de controle e em centros de controle de intoxicações.

\section{REFERÊNCIAS}

1. ALCÂNTARA DA, et al. Intoxicação medicamentosa em criança. Revista Brasileira em Promoção da Saúde, 2013; 16(2): 10-16.

2. ALRUWAILI ND, et al. An epidemiological snapshot of toxicological exposure in children 12 years of age and younger in Riyadh. Annals Of Saudi Medicine, 2019; 39(4): 229-235.

3. AMORIM MLP, at al. Intoxicações em crianças e adolescentes notificados em um centro de toxicologia no nordeste do Brasil. Revista Brasileira de Saúde Materno Infantil, 2017; 17(4): 765-772.

4. BRASIL. Ministério da Saúde. Secretaria de Vigilância em Saúde. Guia de Vigilância em Saúde. 2nd ed. Brasília: Ministério da Saúde; 2017; 705 p.

5. BRITO JG, MARTINS CBG. Intoxicação acidental na população infantojuvenil em ambiente domiciliar: perfil dos atendimentos de emergência. Revista da Escola de Enfermagem da USP, 2015; 49(3): 372-379.

6. DATASUS. 2019. In: Sistema de Informação de Agravos de Notificação (SINAN). Brasília: Ministério da Saúde. Disponível em: http://www2.datasus.gov.br/DATASUS/index.php?area=02. Acesso em: 21 dez. 2019.

7. DOMINGOS SM, et al. Internações por intoxicação de crianças de zero a 14 anos em hospital de ensino no Sul do Brasil, 2006-2011. Epidemiologia e Serviços de Saúde, 2016; 25(2): 1-2. 
8. GUIMARÃES TRA, et al. Perfil epidemiológico das vítimas de intoxicação exógena em Porto Nacional (TO) no período de 2013 a 2017. ScireSalutis, 2019; 9(2): 37-48.

9. LACERDA EMCB, et al. Perfil epidemiológico dos casos de intoxicação notificados no Estado do Maranhão. Revista de Investigação Biomédica, 2018; 9(2): 129-137.

10. LADEIRA RM, et al. Prevenção de intoxicações acidentais domiciliares em crianças: o que diz a literatura? Revista Medica de Minas Gerais, 2016; 26(8): 376-380.

11. LOURENÇO J, et al. Exogenic poisoning in children assisted in a pediatric emergency unit. Acta Paulista de Enfermagem, 2008; 21(2): 282-286.

12. MADDEN M. PediatricToxicology: Emerging Trends. Journal Of Pediatric Intensive Care, 2015; 4(2): 103-110.

13. MAGALHÃES JV, et al. Caracterização das intoxicações medicamentosas registradas no centro de informações toxicológicas do Piauí no período de 2007 a 2012. Revista de Pesquisa Cuidado é Fundamental Oline, 2013; 5(6): 5563.

14. MAIO MC et al. Cor/raça no Estudo Pró-Saúde: resultados comparativos de dois métodos de autoclassificação no Rio de Janeiro, Brasil. Cad. Saúde Pública, Rio de Janeiro, 21(1):171-180, jan-fev, 2005.

15. MARTINS CBG, et al. Envenenamentos acidentais entre menores de 15 anos em município da Região Sul do Brasil. Cadernos de Saúde Pública, 2006; 22(2): 407-414.

16. MORADI M, et al. A hospital base epidemiology and pattern of acute adult poisoning across Iran: a systematic review. Electronic Physician, 2016; 8(9): 2860-2870.

17. OLIVEIRA FFS, SUCHARA EA. Perfil epidemiológico das intoxicações exógenas em crianças e adolescentes em município do mato grosso. Revista Paulista de Pediatria, 2014; 32(4): 299-305.

18. SILVA MF, et al. Determining factors of domestic accidents in early childhood. Journal Of Human Growth And Development, 2017; 27 (1), 10-18.

19. SILVA TJ, OLIVEIRA VB. Intoxicação Medicamentosa Infantil no Paraná. Visão Acadêmica, 2018; 1(19): 51-61.

20. SIQUEIRA KM, et al. Perfil das intoxicações exógenas infantis atendidas em um hospital especializado da rede pública de Goiânia-GO. Revista Eletrônica de Enfermagem, 2008; 10(3): 662-672.

21. SOWMYA S, et al. Poisoning in children: Experience at a tertiary care hospital in Mangalore. International Journal Of Medical Science And Public Health, 2014; 3(11): 1418-1420.

22. TAVARES EO, et al. Fatores associados à intoxicação infantil. Escola Anna Nery, 2013; 17(1): 31-37.

23. VILAÇA L, CARDOSO P. Poisoning among children: an overview of the profile of poisonings in different countries. Revista Médica de Minas Gerais, 2014; 24(1): 21-25.

24. VILAÇA L, et al. Intoxicações exógenas acidentais em crianças e adolescentes atendidos em um serviço de toxicologia de referência de um hospital de emergência brasileiro. Revista Paulista de Pediatria, 2020; 38, 1-8.

25. WORLD HEALTH ORGANIZATION (WHO). 2018. In: Poisoning prevention and management. World Health Organization. Disponível em: https://www.who.int/ipcs/poisons/en/. Acesso em 18 dez. 2019.

26. WORLD HEALTH ORGANIZATION (WHO). World report on child injury prevention. Geneva: World Health Organization; 2008; $211 \mathrm{p}$. 